ULM-TP/06-1

July 2006

\title{
The Selberg trace formula for Dirac operators
}

\author{
Jens Bolte ${ }^{1}$ and Hans-Michael Stiepan ${ }^{2}$ \\ Abteilung Theoretische Physik \\ Universität Ulm, Albert-Einstein-Allee 11 \\ D-89069 Ulm, Germany
}

\begin{abstract}
We examine spectra of Dirac operators on compact hyperbolic surfaces. Particular attention is devoted to symmetry considerations, leading to non-trivial multiplicities of eigenvalues. The relation to spectra of Maaß-Laplace operators is also exploited. Our main result is a Selberg trace formula for Dirac operators on hyperbolic surfaces.
\end{abstract}

\footnotetext{
${ }^{1}$ E-mail address: jens.bolte@uni-ulm.de

${ }^{2}$ E-mail address: hans-michael.stiepan@uni-ulm.de
} 


\section{Introduction}

Trace formulae play a prominent role in spectral geometry and in quantum chaos. In spectral geometry they relate spectra of certain (pseudo-) differential operators on manifolds to the geometry of that manifold. The prime example of such a trace formula, the classical Selberg trace formula Sel56], is concerned with the Laplace-Beltrami operator on a hyperbolic manifold, i.e., a Riemannian manifold with constant negative sectional curvatures. This trace formula establishes a connection between the spectrum of the Laplacian and the length spectrum of closed geodesics on the manifold.

In a semiclassical context spectra of semiclassical operators are related to the periodic orbits of an associated Hamiltonian flow. In extensions of this procedure to operators acting on sections in vector bundles over the relevant phase space, it turned out that the Hamiltonian flow does not provide the entire input on the classical side of the trace formula (see, e.g., BK99]). In particular, for the Dirac operator on $\mathbb{R}^{3}$ the corresponding classical dynamics are given in terms of skew-product flows over Hamiltonian flows, with spin precessions as their cocycles. A similar result is found in the case of a trace formula for the Dirac operator on a graph BH03]. Very recently, in the context of quantum ergodicity Jakobson and Strohmaier [JS06] observed that (the square of) a Dirac operator on a compact Riemannian manifold is related to the associated frame flow, which is a geometric analogue of the skew-product flows in the semiclassical setting.

Here we introduce Dirac operators on compact hyperbolic surfaces, investigate their spectra in terms of symmetries, and finally develop the related Selberg trace formula in a classical setting. This is a direct approach employing Green's functions and pointpair invariants, and therefore complements previous work [BO95], where trace formulae for positive square-roots of squared Dirac operators were determined using Lie-algebraic methods. On the way we exploit the well-known relation of the squared Dirac operator to a Laplacian that contains a coupling to a magnetic field (see, e.g., Pnu94]). In particular, we show that spectra of these Maaß-Laplacians, when they correspond to odd weights, possess multiplicities of at least two. This is a consequence of Kramers' degeneracy in the spectrum of the Dirac operator. The geometric side of the trace formula for the Dirac operator is primarily determined by the closed geodesics on the surface. In addition, we identify traces of the frame flow and its lift into the spin structure, which provide suitable phase factors associated with the closed geodesics.

The paper is organised as follows: In Section 2 we review the definition of a Dirac operator on a Riemannian manifold and explicitly carry out the constructions in the case of the hyperbolic plane. Section 3 then is devoted to an identification of spinor bundles over compact hyperbolic surfaces via factors of automorphy. We then investigate symmetries of the Dirac operator on a compact surface and study their influence on the spectrum in Section 4. After having introduced point-pair invariants in Section 5 we calculate traces of Hilbert-Schmidt operators to arrive at the desired trace formula in Section 6. Finally, in Section [7, we discuss some applications. 


\section{The Dirac operator}

A Dirac operator is a first order, elliptic differential operator acting on sections in a spinor bundle over a Riemannian manifold. For its construction one first needs to supply an orientable, $n$-dimensional Riemannian manifold $(M, \mathbf{g})$ with a spin structure. To this end, let $F M$ be the $\mathrm{SO}(n)$-principal bundle of oriented orthonormal frames of $T M$. A Spin $(n)$ principal bundle $Q$ is called spin structure on $M$ if there exists a principal-bundle morphism $\phi: Q \rightarrow F M$ that is equivariant with respect to the two-fold covering $\Lambda: \operatorname{Spin}(n) \rightarrow \operatorname{SO}(n)$ (see e.g. [Fri97, Bau81]). Moreover, in even dimensions $n$ the complexified Clifford algebra $\mathfrak{C}_{n}^{c}$ is isomorphic to $\mathrm{M}\left(2^{n / 2}, \mathbb{C}\right)$, i.e., the algebra of complex $2^{n / 2} \times 2^{n / 2}$ matrices. The action of $\mathrm{M}\left(2^{n / 2}, \mathbb{C}\right)$ on $\mathbb{C}^{2^{n / 2}}$ defines the Clifford module $\Delta_{n}$. As $\operatorname{Spin}(n)$ is contained in $\mathfrak{C}_{n}^{c}, \Delta_{n}$ is also a module for the spin group. This yields the spin representation $\rho: \operatorname{Spin}(n) \rightarrow$ $\operatorname{Aut}\left(\Delta_{n}\right)$, which can be used to associate the spinor bundle $S:=Q \times{ }_{\rho} \Delta_{n}$ to a given spin structure.

The definition of the Dirac operator $\mathrm{D}$ rests on the canonical connection on $Q$ that is given by the lift of the Levi-Civita connection on $F M$. Denoting the space of smooth sections of $S$ by $C^{\infty}(S)$, this connection induces a covariant derivative $\nabla^{S}: C^{\infty}(S) \rightarrow$ $C^{\infty}\left(S \otimes T^{*} M\right)$. One then defines $\mathrm{D}=\mu \circ \iota \circ \nabla^{S}$, where $\iota$ denotes the canonical isomorphism between $T^{*} M$ and $T M$ induced by the metric $\mathbf{g}$, and $\mu$ is the Clifford multiplication of a vector field and a smooth section in $S$.

The space $C_{0}^{\infty}(S)$ of smooth, compactly supported sections in $S$ can be turned into a pre-Hilbert space by introducing the inner product

$$
\langle\Psi, \Phi\rangle_{\mathrm{L}^{2}}:=\int_{M}\langle\Psi(m), \Phi(m)\rangle_{\mathbb{C}^{2 n / 2}} \mathrm{~d} \mu(m)
$$

where $\mathrm{d} \mu(m)$ denotes the volume form on $M$. The resulting Hilbert space will be denoted by $\mathrm{L}^{2}(S)$. The Dirac operator D is elliptic and essentially self-adjoint on $C_{0}^{\infty}(S)$, thus its spectrum is real. Moreover, on a compact manifold $M$ the spectrum is discrete.

In the sequel we will focus on compact surfaces of constant negative curvature. Their universal covering space is the upper half-plane $\mathbb{H}^{2}:=\left\{(x, y) \in \mathbb{R}^{2} \mid y>0\right\}$ endowed with the Poincaré metric $\mathbf{g}=y^{-2}(\mathrm{~d} x \otimes \mathrm{d} x+\mathrm{d} y \otimes \mathrm{d} y)$ of constant negative Gaussian curvature

$K=-1$. The group of orientation-preserving isometries $\operatorname{PSL}(2, \mathbb{R})=\operatorname{SL}(2, \mathbb{R}) /\{ \pm \operatorname{Id}\}$ acts on $\mathbb{H}^{2}$ via fractional linear transformations,

$$
z \mapsto \gamma z=\frac{a z+b}{c z+d},
$$

where $\gamma=\left(\begin{array}{ll}a & b \\ c & d\end{array}\right) \in \mathrm{SL}(2, \mathbb{R})$ is a representative for an element in $\operatorname{PSL}(2, \mathbb{R})$ and $z=x+\mathrm{i} y$ is viewed as a point in $\mathbb{H}^{2}$. Any compact hyperbolic surface can now be represented as $\Gamma \backslash \mathbb{H}^{2}$, where $\Gamma \subset \operatorname{PSL}(2, \mathbb{R})$ is a discrete, strictly hyperbolic subgroup (a cocompact Fuchsian group of the first kind).

On the simply connected upper half-plane the relevant bundles are trivial, i.e., $F \mathbb{H}^{2} \simeq$ $\mathbb{H}^{2} \times \operatorname{SO}(n)$ and $Q \simeq \mathbb{H}^{2} \times \operatorname{Spin}(n)$. This allows an immediate construction of the Dirac 
operator, which is explicitly given as the matrix-valued operator

$$
\mathrm{D}=\mathrm{i}\left(\begin{array}{cc}
0 & \mathrm{i} y \frac{\partial}{\partial x}+y \frac{\partial}{\partial y}-\frac{1}{2} \\
-\mathrm{i} y \frac{\partial}{\partial x}+y \frac{\partial}{\partial y}-\frac{1}{2} & 0
\end{array}\right),
$$

see, e.g., also [Pnu94]. The operators appearing in the off-diagonals have been introduced by Maaß and have been further investigated by Roelcke ( Maa53, Roe66, Roe67]). In fact, they considered the operators

$$
\begin{aligned}
\mathrm{K}_{k} & :=\mathrm{i} y \frac{\partial}{\partial x}+y \frac{\partial}{\partial y}+\frac{k}{2} \\
\Lambda_{k} & :=\mathrm{i} y \frac{\partial}{\partial x}-y \frac{\partial}{\partial y}+\frac{k}{2} .
\end{aligned}
$$

It is therefore natural to introduce the weighted Dirac operators

$$
\mathrm{D}_{k}:=\mathrm{i}\left(\begin{array}{cc}
0 & \mathrm{~K}_{k-2} \\
-\Lambda_{k} & 0
\end{array}\right)
$$

which reduce to the Dirac operator for $k=1$. In physical terms the additional parameter $k$, which from now on will be called a weight, corresponds to a constant magnetic field on the surface. In normalised units the field strength is given by

$$
B=\frac{k-1}{2 e}
$$

On a compact surface the Dirac quantisation condition for the magnetic flux implies that $k$ must be an integer. Therefore, from now on we will only consider $k \in \mathbb{Z}$.

The weighted Dirac operator $\mathrm{D}_{k}$ is closely related to the weighted Maaß-Laplacian,

$$
\Delta_{k}=-\mathrm{K}_{k-2} \Lambda_{k}-\frac{k}{2}\left(1-\frac{k}{2}\right)=y^{2}\left(\frac{\partial^{2}}{\partial x^{2}}+\frac{\partial^{2}}{\partial y^{2}}\right)-\mathrm{i} k y \frac{\partial}{\partial x},
$$

since

$$
\mathrm{D}_{k}^{2}=\left(\begin{array}{cc}
-\Delta_{k}-\frac{k}{2}\left(1-\frac{k}{2}\right) & 0 \\
0 & -\Delta_{k-2}-\frac{k}{2}\left(1-\frac{k}{2}\right)
\end{array}\right) .
$$

We remark that both the weighted Laplacians and the weighted Dirac operators are elliptic operators as their principal symbols do not depend on $k$.

As yet we have neither specified spaces of sections in spinor bundles $S$, nor have we introduced Dirac operators on compact surfaces. It turns out that both purposes can be conveniently achieved by identifying suitable automorphic forms. We devote the following section to this task.

\section{Automorphic forms}

All relevant bundles over a compact surface $\Gamma \backslash \mathbb{H}^{2}$ correspond to trivial bundles over $\mathbb{H}^{2}$ (see Gun56, Gun76]). More specifically, given the canonical projection $p: \mathbb{H}^{2} \rightarrow \Gamma \backslash \mathbb{H}^{2}$ 
and the vector bundle $\zeta=\left(E, \Gamma \backslash \mathbb{H}^{2}, \pi, V\right)$ over the compact surface, its pullback $p^{*}(\zeta)$ is trivial, i.e., the total space is isomorphic to $\mathbb{H}^{2} \times V$. Now any covering translation $\gamma \in \Gamma$ extends to a bundle homomorphism $\tilde{\gamma}$ on $p^{*}(\zeta)$, and this can be written as

$$
\tilde{\gamma}(z, v)=(\gamma z, \sigma(z, \gamma) v)
$$

where $\sigma: \mathbb{H}^{2} \times \Gamma \rightarrow \mathrm{GL}(V)$.

For our purposes it will be useful to consider the subgroup $\bar{\Gamma} \subset \operatorname{SL}(2, \mathbb{R})$ that corresponds to the Fuchsian group $\Gamma \subset \operatorname{PSL}(2, \mathbb{R})$ via $\Gamma=\bar{\Gamma} /\{ \pm \operatorname{Id}\}$. Instead of $\sigma$ one hence considers a function $j: \mathbb{H}^{2} \times \bar{\Gamma} \rightarrow \mathrm{GL}(V)$, called factor of automorphy. To define the action of $\gamma \in \Gamma$ unambiguously one therefore requires a suitable character $\chi$ of $\bar{\Gamma}$ (multiplier system) depending on the value of $j(z,-\mathrm{Id})$. Based on these observations sections in $\zeta$ can be obtained from global sections in $p^{*}(\zeta)$ : any vector-valued function $\psi: \mathbb{H}^{2} \rightarrow V$ with $\psi(\gamma z)=\chi(\gamma) j(z, \gamma) \psi(z)$, called automorphic form, yields a section in $\zeta$, and vice versa.

Definition 1. Let $\Gamma \subset \operatorname{PSL}(2, \mathbb{R})$ be a strictly hyperbolic Fuchsian group, then a multiplier system of weight $k$ is a unitary character $\chi$ of $\bar{\Gamma}$ with $\chi(-\mathrm{Id})=(-1)^{k}$. A factor of automorphy for the Maaß-Laplacian of weight $k$ is defined by $j_{\gamma}(z, k):=\frac{(c z+d)^{k / 2}}{(c \bar{z}+d)^{k / 2}}$ when $\gamma=\left(\begin{array}{ll}a & b \\ c & d\end{array}\right)$. Then $\psi: \mathbb{H}^{2} \rightarrow \mathbb{C}$ is an automorphic form for the Maaß-Laplacian of weight $k$, if

$$
\psi(\gamma z)=\chi(\gamma) j_{\gamma}(z, k) \psi(z), \quad \forall \gamma \in \bar{\Gamma} .
$$

The space of all such automorphic forms is denoted by ${ }^{L} \mathfrak{F}(\Gamma, k, \chi)$. Similarly, ${ }^{L} \mathfrak{C}^{l}(\Gamma, k, \chi)$ and ${ }^{L} \mathfrak{L}^{2}(\Gamma, k, \chi)$ are the spaces of differentiable and square-integrable automorphic forms, respectively. In the latter case, integrability is meant with respect to the measure $\mathrm{d} \mu(z)=$ $\frac{\mathrm{d} x \mathrm{~d} y}{y^{2}}$ over a fundamental domain $F \subset \mathbb{H}^{2}$ of $\Gamma$.

It is well-known that $\Delta_{k}$ acting on a (twice differentiable) automorphic form of weight $k$ yields again an automorphic form of weight $k$ (see [Roe66, Roe67, Hej76]). We denote the space of all eigenforms of $-\Delta_{k}$ with eigenvalue $\lambda$ by $L_{\mathfrak{F}_{\lambda}}(\Gamma, k, \chi)$. In case $k \geq 2$ there exist certain special eigenvalues that are known explicitly,

$$
\lambda_{j}^{(k)}=\frac{k-2 j}{2}\left(1-\frac{k-2 j}{2}\right), \quad j=0,1,2, \ldots,\left[\frac{k-1}{2}\right],
$$

where $[x]$ is the integer part of $x \in \mathbb{R}$. For multiplier systems $\chi \not \equiv 1$ the eigenvalue (3.2) has a multiplicity $(g-1)(k-2 j-1)$, see Hej76]; here $g \geq 2$ is the genus of the closed surface $\Gamma \backslash \mathbb{H}^{2}$.

The relations (2.1) and (2.3) now suggest the following definition of automorphic forms for the weighted Dirac operator.

Definition 2. Let

$$
J_{\gamma}(z, k):=\left(\begin{array}{cc}
j_{\gamma}(z, k) & 0 \\
0 & j_{\gamma}(z, k-2)
\end{array}\right)
$$


Then we define the space $\mathfrak{F}(\Gamma, k, \chi)$ of automorphic forms for the Dirac operator with weight $k$ to consist of the functions $\Psi: \mathbb{H}^{2} \rightarrow \Delta_{2}=\mathbb{C}^{2}$ that transform as

$$
\Psi(\gamma z)=\chi(\gamma) J_{\gamma}(z, k) \Psi(z), \quad \forall \gamma \in \bar{\Gamma}
$$

The spaces $\mathfrak{C}^{l}(\Gamma, k, \chi), \mathfrak{L}^{2}(\Gamma, k, \chi)$ are defined analogously, and $\mathfrak{F}_{\rho}(\Gamma, k, \chi)$ denotes the eigenspace of $-\mathrm{D}_{k}$ corresponding to the eigenvalue $\rho$.

In order to demonstrate that the weighted Dirac operator $\mathrm{D}_{k}$ maps a differentiable automorphic form of the type (3.3) to a form of the same type, one has to show that under a covering translation $\gamma$ the operator $\mathrm{D}_{k}$ behaves as

$$
\mathrm{D}_{k} \mapsto J_{\gamma}(z, k) \mathrm{D}_{k} J_{\gamma}^{-1}(z, k) .
$$

This can be done in a straightforward calculation. Hence, the automorphic forms (3.3) represent sections in spinor bundles over the surface $\Gamma \backslash \mathbb{H}^{2}$.

We also note that $\mathrm{D}_{k}$ is essentially self-adjoint on $\mathfrak{C}^{1}(\Gamma, k, \chi)$. The proof is practically identical to the case of the Dirac operator $(k=1)$, which is well-known (see, e.g., [Fri97] where also Spin ${ }^{\mathbb{C}}$-connections are considered). Thus we conclude that the spectrum of $\mathrm{D}_{k}$ is real and discrete.

We are now able to establish a connection between the eigenforms of the Laplacian and of the Dirac operator. For this we recall that the spectrum of $-\Delta_{k}$ is bounded from below by $\frac{k}{2}\left(1-\frac{k}{2}\right)$ Roe66].

Proposition 1. Let $\Psi=\left(\begin{array}{c}\psi_{1} \\ \psi_{2}\end{array}\right)$ be an eigenform of $-\mathrm{D}_{k}$ with eigenvalue $\rho$, and let $\lambda=$ $\rho^{2}+\frac{k}{2}\left(1-\frac{k}{2}\right)$. Then $\psi_{1} \in L_{\mathfrak{F}_{\lambda}}(\Gamma, k, \chi)$. On the other hand, if $\psi \in L_{\mathfrak{F}_{\lambda}}(\Gamma, k, \chi)$, then $\Psi=$ $\left(\begin{array}{c}\rho \psi \\ \mathrm{i} \Lambda_{k} \psi\end{array}\right) \in \mathfrak{F}_{\rho}(\Gamma, k, \chi)$. Moreover, if eigenforms of the Dirac operator $(\rho \neq 0)$ are linearly independent, the same holds for the corresponding eigenforms of the Maaß-Laplacian, and vice versa.

Proof. As both operators are elliptic all eigenforms are smooth. The relation between $\rho$ and $\lambda$ can be obtained by using eq. (2.3). To establish the desired transformation properties one has to write down the conditions on the components. The rest then follows from [Roe66]. The linear independence can be obtained from some straight-forward manipulations using

$$
-\mathrm{i} \Lambda_{k} \psi_{1}+\rho \psi_{2}=0
$$

which can be solved for $\psi_{2}$ if $\rho \neq 0$.

\section{Symmetries}

Apart from the fact that spectra of weighted Dirac operators on compact surfaces are real and discrete, further spectral properties can be concluded from symmetry considerations. 
Lemma 1. The weighted Dirac operator possesses a chiral symmetry, i.e., if $\Psi=\left(\begin{array}{c}\psi_{1} \\ \psi_{2}\end{array}\right) \in$ $\mathfrak{F}_{\rho}(\Gamma, k, \chi)$, then $\left(\begin{array}{c}\psi_{1} \\ -\psi_{2}\end{array}\right) \in \mathfrak{F}_{-\rho}(\Gamma, k, \chi)$. Hence the spectrum of $\mathrm{D}_{k}$ is symmetric about zero.

Proof. The proof amounts to a simple calculation which can be performed conveniently by using the relations in [Roe66] once again.

The symmetry statement extends to zero modes in that the Atiyah-Singer index theorem (see Hit74, AS68) implies that their number is even. From now on we will denote this number by $2 N$.

A further symmetry is concerned with quantum mechanical time-reversal. For the Dirac operators considered here this is implemented through the anti-linear operator $\mathrm{T}:=\mathrm{i} \sigma_{2} \mathrm{C}$ acting on automorphic forms of arbitrary weight, where $\mathrm{C}$ denotes complex conjugation and $\sigma_{2}=\left(\begin{array}{cc}0 & -\mathrm{i} \\ \mathrm{i} & 0\end{array}\right)$ is one of the Pauli matrices. Hence $\mathrm{T}^{2}=-\mathrm{Id}$. Moreover, on the Hilbert space $\mathfrak{L}^{2}(\Gamma, k, \chi)$ this operator is anti-unitary.

Lemma 2. Let $\Psi \in \mathfrak{F}_{\rho}(\Gamma, k, \chi)$, then $\mathrm{T} \Psi \in \mathfrak{F}_{\rho}(\Gamma, 2-k, \bar{\chi})$. In particular, if $k=1$ and the multiplier system $\chi$ is real-valued every eigenvalue of $\mathrm{D}$ has a multiplicity of (at least) two.

Proof. The first part of this lemma can be checked by straight-forward calculations that we omit here. For the second part one merely has to use the fact that $\mathrm{T}$ is anti-unitary with $\mathrm{T}^{2}=-\mathrm{Id}$. This immediately yields that the eigenforms $\Psi$ and T $\Psi$ with eigenvalue $\rho$ are linearly independent.

At this point we add a few remarks:

(i) Above we have always assumed that $\Gamma$ contains, apart from unity, only hyperbolic elements; hence there are no elements of finite order. Thus one can construct a realvalued multiplier system by simply assigning \pm 1 to the generators of $\Gamma$ and extending this to $\bar{\Gamma}$. If one also allows for elliptic elements this clearly will not work.

(ii) Lemma 2 says that $\mathrm{D}_{k}$ and $\mathrm{D}_{2-k}$ possess identical non-vanishing eigenvalues (including multiplicities). According to (2.2) the weights $k$ and $2-k$, up to sign correspond to the same magnetic field strengths. We will therefore restrict subsequent discussions to the case $k \geq 1$.

(iii) In general, quantum systems with half-integer spin share the same behaviour under time-reversal as in the present case. For the respective quantum Hamiltonians the multiplicity two of their eigenvalues due to time-reversal symmetry is known as Kramers' degeneracy Kra30. Moreover, magnetic fields usually break time-reversal invariance. This is the reason for the special role played by the weight $k=1$.

(iv) In his tenfold-way scheme Zirnbauer classified quantum systems according to their behaviour under basic symmetry operations like time-reversal, rotations, and chiral transformations Zir96. The resulting symmetry classes are unambiguously linked with Cartan's ten classes of symmetric spaces. In this context the present case, with time-reversal symmetry, $\mathrm{T}^{2}=-\mathrm{Id}$, and chiral symmetry is identified as the type $C \mathrm{II}$ and is related to the symmetric space $\operatorname{Sp}(p+q) / \operatorname{Sp}(p) \times \operatorname{Sp}(q)$ (of compact type). 
(v) Following the conjecture of Bohigas, Giannoni, and Schmit BGS84 one expects that, generically, correlations among the eigenvalues of geometric operators on manifolds of negative curvature can be described by random matrix theory. (See, however, exceptions when the Fuchsian group is arithmetic [BGGS92, BSS92.) The relevant random matrix ensemble for the present case is the chiral Gaussian symplectic ensemble (chGSE).

From supersymmetry considerations (see, e.g., Tha92]) it is known that eigenvalues of the square of a Dirac operator have even multiplicities. We stress that this observation is unrelated to Lemma 2] Rather, this degeneracy stems from chiral symmetry (Lemma 1) combined with the squaring.

However, the degeneracy due to time reversal somewhat unexpectedly extends to the spectrum of $\mathrm{D}_{k}$ for odd weight. To this end we recall that the Maaß operator $\mathrm{K}_{k}$ raises the weight by two, without changing the eigenvalue of an eigenform of the appropriate MaaßLaplacian [Roe66, Roe67]. It hence maps ${ }^{L} \mathfrak{F}_{\lambda}(\Gamma, k, \chi)$ to ${ }^{L} \mathfrak{F}_{\lambda}(\Gamma, k+2, \chi)$. The operator $\Lambda_{k+2}$ is its formal adjoint from ${ }^{L} \mathfrak{L}^{2}(\Gamma, k+2, \chi)$ to ${ }^{L} \mathfrak{L}^{2}(\Gamma, k, \chi)$. As a consequence, up to the special eigenvalues (3.2) the spectrum of $-\Delta_{k}$ depends only on $k \bmod 2$.

Lemma 3. Let $\Gamma$ be a strictly hyperbolic group and $\chi$ a real-valued multiplier system. If the weight $k$ is odd the eigenvalues of the Maaß-Laplacian $\Delta_{k}$ have a multiplicity of (at least) two.

Proof. For odd $k=2 m+1$, the multiplicities of the special eigenvalues (3.2) are known to be $(g-1) 2(m-j)$, see Hej76 and below equation (3.2). In all other cases the statement follows immediately from Proposition 1 and Lemma 2.

Apart from the special eigenvalues, a twofold degeneracy of the eigenvalues can also be obtained in a constructive way. To this end, one first lowers the weight from $k=$ $2 m+1, m \in \mathbb{N}_{0}$, to one by successive applications of operators $\Lambda_{l}$, then applies complex conjugation (i.e., time reversal for spin zero), and finally raises the weight back to $k=$ $2 m+1$. Altogether, one thus applies the anti-linear operator

$$
{ }^{L} S_{2 m+1}:=\mathrm{C} \Lambda_{-2 m+1} \Lambda_{-2 m+3} \ldots \Lambda_{2 m+1} .
$$

Now some straight-forward calculations show that this way an eigenform $\psi$ is mapped to a linearly independent eigenform $\phi={ }^{L} S_{2 m+1} \psi$ of the same weight and with the same eigenvalue. In particular, $\phi$ may only vanish when its eigenvalue is of the special type (3.2).

We stress that this argument fails if $k$ is even. In the case $k=0$ this is easy to see: if $\psi \in L_{\mathfrak{F}}(\Gamma, 0, \chi)$, then $\mathrm{C} \psi \in L_{\mathfrak{F}_{\rho}}(\Gamma, 0, \chi)$ as long as $\chi$ is real. But it is well-known that for $k=0$ the eigenforms can be chosen to be real-valued, so $\psi$ and $\mathrm{C} \psi$ are not linearly independent.

Of course, according to Proposition 1 the degeneracy in the spectra of Maaß-Laplacians implies a corresponding degeneracy in the spectra of the Dirac operators $\mathrm{D}_{k}$. Except for 
the eigenvalues that derive from the special eigenvalues (3.2) of the associated MaaßLaplacian, one can again devise a constructive approach which reveals more clearly that the degeneracy is a consequence of a 'generalised time reversal symmetry'. To this end we introduce the operator

$$
\mathrm{A}_{k}^{\dagger}:=\left(\begin{array}{cc}
\rho^{\prime} \mathrm{K}_{k} & 0 \\
\mathrm{i} k & \rho \mathrm{K}_{k-2}
\end{array}\right) .
$$

This maps $\mathfrak{F}_{\rho}(\Gamma, k, \chi)$ into $\mathfrak{F}_{\rho^{\prime}}(\Gamma, k+2, \chi)$, where $\rho^{\prime}$ is determined by $\rho^{2}+k=\rho^{\prime 2}$ and $\operatorname{sgn}\left(\rho^{\prime}\right)=\operatorname{sgn}(\rho)$. From [Roe66, Satz 5.3] one can deduce that $\mathrm{A}_{k}^{\dagger}$ indeed is an isomorphism between $\mathfrak{F}_{\rho}(\Gamma, k, \chi)$ and $\mathfrak{F}_{\rho^{\prime}}(\Gamma, k+2, \chi)$ if $\rho \neq 0$. Its formal adjoint reads

$$
\mathrm{A}_{k+2}:=-\left(\begin{array}{cc}
\rho^{\prime} \Lambda_{k+2} & \mathrm{i} k \\
0 & \rho \Lambda_{k}
\end{array}\right)
$$

The analogue to the operator (4.1) now is

$$
S_{2 m+1}:=\mathrm{B}_{2 m-1}^{\dagger} \ldots \mathrm{B}_{1}^{\dagger} \mathrm{TC}_{3} \ldots \mathrm{C}_{2 m+1}
$$

where $\mathrm{B}_{k}^{\dagger}:=\frac{1}{\rho} \mathrm{A}_{k}^{\dagger}$ and $\mathrm{C}_{k}=\frac{1}{\rho^{\prime}} \mathrm{A}_{k}$. A direct calculation shows that $\mathrm{S}_{2 m+1}$ does not depend on $\rho$.

Proposition 2. Let $k=2 m+1$ and assume that the multiplier system $\chi$ is real-valued. Then the eigenvalues of the weighted Dirac operator $\mathrm{D}_{2 m+1}$ occur with multiplicities of at least two. Moreover,

$$
\mathrm{S}_{2 m+1}=\mathrm{T}\left(\begin{array}{cc}
\Lambda_{-2 m+3} \Lambda_{-2 m+5} \ldots \Lambda_{2 m+1} & 0 \\
0 & \Lambda_{-2 m+1} \Lambda_{-2 m+3} \ldots \Lambda_{2 m-1}
\end{array}\right)
$$

is identical on all spaces $\mathfrak{F}_{\rho}(\Gamma, 2 m+1, \chi)$ of eigenforms, and can therefore be extended to all of $\mathfrak{C}^{\infty}(\Gamma, 2 m+1, \chi)$. Then $\Psi \in \mathfrak{F}_{\rho}(\Gamma, 2 m+1, \chi)$ implies $\Phi:=\mathrm{S}_{2 m+1} \Psi \in \mathfrak{F}_{\rho}(\Gamma, 2 m+1, \chi)$ and $\langle\Phi, \Psi\rangle_{\mathrm{L}^{2}}=0$. For $m>0$ the form $\Phi$ vanishes identically iff $\rho$ is related to one of the special eigenvalues (3.2) via $\lambda_{j}^{(k)}=\rho^{2}+\frac{k}{2}\left(1-\frac{k}{2}\right)$.

Proof. The statement about the multiplicities of eigenvalues is implied by Proposition [1] and Lemma 3. The conclusions $\Phi \in \mathfrak{F}_{\rho}(\Gamma, k, \chi)$ and $\langle\Phi, \Psi\rangle_{\mathrm{L}^{2}}=0$ follow directly from the preceding considerations. One must only take care of the possibility that $\Phi$ may vanish. But evaluating $\|\Phi\|_{\mathrm{L}^{2}}$ shows that this norm is zero, iff $\lambda=\rho^{2}+\frac{k}{2}\left(1-\frac{k}{2}\right)$ is of the form (3.2).

\section{Point-pair invariants}

From the case of the Laplacian it is well known that setting up a Selberg trace formula amounts to calculating traces of Hilbert-Schmidt operators L that commute with the Laplacian Sel56, Hej76, Hej83]. The kernels of such operators can be obtained from suitable 
Poincaré series over so-called point-pair invariants, whose construction will be briefly outlined in this section.

We first introduce two matrix-valued functions on the upper half-plane, which help to study transformation properties under fractional linear transformations,

$$
\begin{aligned}
& A\left(z^{\prime}, z\right):=\left(\begin{array}{cc}
\frac{\left(z-\bar{z}^{\prime}\right)^{\frac{1}{2}}}{\left(z^{\prime}-z\right)^{\frac{1}{2}}} & 0 \\
0 & \frac{\left(z^{\prime}-\bar{z}\right)^{\frac{1}{2}}}{\left(\bar{z}-\bar{z}^{\prime}\right)^{\frac{1}{2}}}
\end{array}\right), \\
& B\left(z^{\prime}, z\right):=\left(\begin{array}{cc}
\frac{\left(z^{\prime}-z\right)^{\frac{1}{2}}}{\left(z^{\prime}-\bar{z}\right)^{\frac{1}{2}}} & 0 \\
0 & \frac{\left(\bar{z}-\bar{z}^{\prime}\right)^{\frac{1}{2}}}{\left(z-\bar{z}^{\prime}\right)^{\frac{1}{2}}}
\end{array}\right) .
\end{aligned}
$$

Definition 3. Let $\Phi=\left(\begin{array}{cc}\Phi_{1} & \Phi_{2} \\ \Phi_{3} & \Phi_{4}\end{array}\right):[1, \infty) \rightarrow \mathbb{R}^{2 \times 2}$ be continuous. Assume moreover that $\Phi_{2}=\Phi_{3}$ and that there exists a constant $C_{\epsilon}$ such that each component satisfies

$$
\left|\Phi_{i}(\sigma)\right| \leq C_{\epsilon} \sigma^{-1-\epsilon}
$$

for some $\epsilon>0$. Then we call

$$
K\left(z^{\prime}, z\right)=-\mathrm{i} A\left(z^{\prime}, z\right) \Phi\left(\frac{1}{2}\left(\cosh \left(d\left(z^{\prime}, z\right)+1\right)\right) B\left(z^{\prime}, z\right),\right.
$$

where $d\left(z^{\prime}, z\right)$ denotes the hyperbolic distance of two points $z, z^{\prime} \in \mathbb{H}^{2}$, a point-pair invariant.

Applying any transformation $\gamma \in \mathrm{SL}(2, \mathbb{R})$ yields

$$
K\left(\gamma z^{\prime}, \gamma z\right)=J_{\gamma}\left(z^{\prime}, 1\right) K\left(z^{\prime}, z\right) J_{\gamma}^{-1}(z, 1) .
$$

The restrictions on the components of $\Phi$ assure that $K$ is hermitian, i.e., $K\left(z^{\prime}, z\right)=$ $K^{\dagger}\left(z, z^{\prime}\right)$. In order to proceed we need a technical Lemma, which can be found in Hej76.

Lemma 4. Let $f:[1, \infty) \rightarrow \mathbb{C}$ and $\epsilon>0$. Moreover, assume that

$$
f(\sigma) \leq C_{\epsilon} \sigma^{-1-\epsilon}
$$

holds for all $\sigma$ and a constant $C_{\epsilon}$. Then the sum

$$
\sum_{\gamma \in \bar{\Gamma}} f\left(\frac{1}{2}\left(\cosh \left(d\left(z^{\prime}, \gamma z\right)\right)+1\right)\right)
$$

converges absolutely and uniformly for all $z^{\prime}, z \in \mathbb{H}^{2}$.

Starting from the point-pair invariant we can now construct automorphic kernels via Poincaré series. Due to our restrictions on $\Phi$,

$$
K_{\Gamma}\left(z^{\prime}, z\right)=\frac{1}{2} \sum_{\gamma \in \bar{\Gamma}} K\left(z^{\prime}, \gamma z\right) \chi(\gamma) J_{\gamma}(z, 1)
$$


is well-defined. Some standard manipulations show that the behaviour of $K_{\Gamma}$ under transformations is given by

$$
K_{\Gamma}\left(\gamma_{1} z^{\prime}, \gamma_{2} z\right)=\chi\left(\gamma_{1}\right) J_{\gamma_{1}}\left(z^{\prime}, 1\right) K_{\Gamma}\left(z^{\prime}, z\right) J_{\gamma_{2}}^{-1}(z, 1) \chi^{-1}\left(\gamma_{2}\right)
$$

This allows us to define automorphic kernels.

Definition 4. Let $F$ be a fundamental domain for the Fuchsian group $\Gamma$. We say that $K_{\Gamma}: \mathbb{H}^{2} \times \mathbb{H}^{2} \rightarrow \mathbb{C}^{2 \times 2}$ is an element of $\mathfrak{L}^{2}\left(\Gamma \backslash \mathbb{H}^{2}, \Gamma \backslash \mathbb{H}^{2}, \chi\right)$, if (15.3) holds for all $\gamma \in \bar{\Gamma}$ and

$$
\left\|K_{\Gamma}\right\|_{\mathrm{L}^{2}}^{2}:=\int_{F} \int_{F} \operatorname{tr}\left(K_{\Gamma}^{\dagger}\left(z^{\prime}, z\right) K_{\Gamma}\left(z^{\prime}, z\right)\right) \mathrm{d} \mu(z) \mathrm{d} \mu\left(z^{\prime}\right)<\infty .
$$

It is well-known that such an automorphic kernel defines a Hilbert-Schmidt operator $\mathrm{L}: \mathfrak{L}^{2}(\Gamma, 1, \chi) \rightarrow \mathfrak{L}^{2}(\Gamma, 1, \chi)$ via

$$
[\operatorname{L} \Psi]\left(z^{\prime}\right):=\int_{F} K_{\Gamma}\left(z^{\prime}, z\right) \Psi(z) \mathrm{d} \mu(z) .
$$

If $K_{\Gamma}$ is hermitian there exists a basis $\left\{\Psi_{n}\right\}$ of orthonormal eigenforms of $\mathrm{L}$ in $\mathfrak{L}^{2}(\Gamma, 1, \chi)$ such that $K_{\Gamma}\left(z^{\prime}, z\right)=\sum_{n} a_{n} \Psi_{n}\left(z^{\prime}\right) \Psi_{n}^{\dagger}(z)$. Furthermore, if $K_{\Gamma}$ was constructed from a pointpair invariant via (5.2), then one can easily check that the operator $L$ has a finite trace that is given by $\sum_{n} a_{n}$. In the following section we will construct point-pair invariants from a Green's function and then calculate such traces.

\section{The trace formula}

Before we can proceed to introduce point-pair invariants we have to identify Green's functions for Dirac operators on surfaces $\Gamma \backslash \mathbb{H}^{2}$. For this, and the following, we restrict our attention to the Dirac operator itself, i.e., to the weight $k=1$. The starting point will be Green's function for D on the hyperbolic plane, from which the corresponding Green's function on the compact surface can be obtained in terms of a Poincaré series.

Since the resolvent of $\mathrm{D}$ is a bounded operator when $\operatorname{Im}(\rho)<0$, we can make the ansatz

$$
(\mathrm{D}+\rho)^{-1} \Psi\left(z^{\prime}\right)=\int_{\mathbb{H}^{2}} G\left(z^{\prime}, z ; \rho\right) \Psi(z) \mathrm{d} \mu(z),
$$

with $G(\cdot, \cdot ; \rho): \mathbb{H}^{2} \times \mathbb{H}^{2} \rightarrow \mathbb{C}^{2 \times 2}$. For the matrix entries of $G$ we use the notation $G=$ $\left(\begin{array}{ll}G_{1} & G_{2} \\ G_{3} & G_{4}\end{array}\right)$. Then $G$ can be uniquely characterised as a solution of the matrix-differential equation

$$
(\mathrm{D}+\rho) G\left(z^{\prime}, z ; \rho\right)=0, \quad \text { for } z \neq z^{\prime},
$$

with a specified behaviour in a neighbourhood of $z=z^{\prime}$. That is, for the diagonal matrix entries

$$
\lim _{d\left(z^{\prime}, z\right) \rightarrow 0}\left(G_{i}\left(z^{\prime}, z ; \rho\right)-\frac{\rho}{4 \pi} \log \left(d\left(z^{\prime}, z\right)\right)\right)<\infty, \quad i=1,4
$$


is required, whereas the non-diagonal entries are regular in $z=z^{\prime}$. In addition, $G_{i}\left(z^{\prime}, z ; \rho\right)$ must approach zero as $d\left(z^{\prime}, z\right) \rightarrow \infty$.

Furthermore, the transformation property (3.4) implies the corresponding behaviour

$$
G\left(z^{\prime}, z ; \rho\right)=J_{\gamma}^{-1}\left(z^{\prime}, 1\right) G\left(\gamma z^{\prime}, \gamma z ; \rho\right) J_{\gamma}(z, 1)
$$

of the Green's function under an isometry $\gamma \in \operatorname{PSL}(2, \mathbb{R})$.

In order to solve for this Green's function we closely follow [Roe66, Roe67], where the corresponding problem for the Maaß-Laplacians is treated. We begin with introducing

$$
\check{G}\left(z^{\prime}, z ; \rho\right):=A^{-1}\left(z^{\prime}, z\right) G\left(z^{\prime}, z ; \rho\right) B^{-1}\left(z^{\prime}, z\right),
$$

which is invariant under $\operatorname{PSL}(2, \mathbb{R})$, and note that $\check{\mathrm{D}}:=A^{-1}(z, \mathrm{i}) \mathrm{D} A(z, \mathrm{i})$ is invariant under the stability group $\operatorname{PSO}(2, \mathbb{R})$ of $z=\mathrm{i}$. Therefore, the differential equation for $\check{G}$ corresponding to (6.1) is transformed into polar coordinates $(\sigma, \phi)$ for $z \in \mathbb{H}^{2}$, where $\sigma=\frac{1}{2}\left(\cosh \left(d\left(z, z^{\prime}\right)\right)+1\right)$. With $H(\sigma ; \rho)=\check{G}\left(z^{\prime}, z ; \rho\right)$, this leads to a system of ordinary linear differential equations for $H=\left(\begin{array}{ll}H_{1} & H_{2} \\ H_{3} & H_{4}\end{array}\right)$,

$$
\begin{aligned}
& \left(\begin{array}{cc}
\rho & \mathrm{i}\left[(\sigma(\sigma-1))^{\frac{1}{2}} \frac{\partial}{\partial \sigma}+\frac{1}{2}\left(\frac{\sigma-1}{\sigma}\right)^{\frac{1}{2}}\right] \\
\mathrm{i}\left[(\sigma(\sigma-1))^{\frac{1}{2}} \frac{\partial}{\partial \sigma}+\frac{1}{2}\left(\frac{\sigma-1}{\sigma}\right)^{\frac{1}{2}}\right] & \rho
\end{array}\right) H(\sigma ; \rho) \\
& =-\mathrm{i}\left(\begin{array}{cc}
\frac{1}{2}(\sigma(\sigma-1))^{-\frac{1}{2}} H_{3}(\sigma ; \rho) & 0 \\
0 & \frac{1}{2}(\sigma(\sigma-1))^{-\frac{1}{2}} H_{2}(\sigma ; \rho)
\end{array}\right) .
\end{aligned}
$$

The solution of (6.2) for $\sigma \rightarrow \infty$ is given by

$$
\begin{aligned}
H_{1}(\sigma ; \rho) & =-\frac{\rho}{4 \pi} \sigma^{-\frac{1}{2}-\mathrm{i} \rho} \frac{\Gamma(\mathrm{i} \rho) \Gamma(\mathrm{i} \rho+1)}{\Gamma(2 \mathrm{i} \rho+1)} F\left(\mathrm{i} \rho, 1+\mathrm{i} \rho ; 1+2 \mathrm{i} \rho ; \frac{1}{\sigma}\right) \\
& =-\frac{\rho}{4 \pi} \sigma^{-\frac{1}{2}} \int_{0}^{1} t^{\mathrm{i} \rho}(1-t)^{\mathrm{i} \rho-1}(\sigma-t)^{-\mathrm{i} \rho} \mathrm{d} t
\end{aligned}
$$

and

$$
\begin{aligned}
H_{2}(\sigma ; \rho) & =-\frac{\mathrm{i}}{4 \pi} \sigma^{-1-\mathrm{i} \rho}(\sigma-1)^{\frac{1}{2}} \frac{\Gamma^{2}(\mathrm{i} \rho+1)}{\Gamma(2 \mathrm{i} \rho+1)} F\left(1+\mathrm{i} \rho, 1+\mathrm{i} \rho ; 1+2 \mathrm{i} \rho ; \frac{1}{\sigma}\right) \\
& =\frac{\rho}{4 \pi}(\sigma-1)^{\frac{1}{2}} \int_{0}^{1} t^{\mathrm{i} \rho}(1-t)^{\mathrm{i} \rho-1}(\sigma-t)^{-\mathrm{i} \rho-1} \mathrm{~d} t,
\end{aligned}
$$

where $F(a, b ; c ; z)$ is a hypergeometric function (see, e.g., Erd53). Moreover, $H_{3}(\sigma ; \rho)=$ $H_{2}(\sigma ; \rho)$ and $H_{4}(\sigma ; \rho)=H_{1}(\sigma ; \rho)$. From this representation we infer an upper bound for the components of the Green's function,

$$
\mid G_{i}\left(z^{\prime}, z ; \rho\right) \leq \text { const. }|\rho| \mathrm{e}^{-\left(\frac{1}{2}-\operatorname{Im}(\rho)\right) d\left(z^{\prime}, z\right)}, \quad \text { if } d\left(z^{\prime}, z\right) \geq d_{0}>0 .
$$

As the singularity of $G$ for $d\left(z^{\prime}, z\right) \rightarrow 0$ is integrable we obtain the following Lemma. 
Lemma 5. Let $f: \mathbb{H}^{2} \rightarrow \mathbb{C}^{2}$ be bounded and continuous, then

$$
\int_{\mathbb{H}^{2}} G\left(z^{\prime}, z ; \rho\right) f(z) \mathrm{d} \mu(z)
$$

converges absolutely and uniformly in $\operatorname{Re}(\rho)$ as long as $\operatorname{Im}(\rho)<-\frac{1}{2}$.

With Lemma 4 and the estimate (6.5), Green's function for the Dirac operator on a compact surface can now be obtained in terms of a Poincaré series (5.2).

Lemma 6. Let $\operatorname{Im}(\rho)<-\frac{1}{2}$, then

$$
G_{\Gamma}\left(z^{\prime}, z ; \rho\right):=\frac{1}{2} \sum_{\gamma \in \bar{\Gamma}} G\left(z^{\prime}, \gamma z ; \rho\right) \chi(\gamma) J_{\gamma}(z, 1)
$$

converges for $z \neq z^{\prime} \bmod \Gamma$, and is the Green's function for $\mathrm{D}$ on $\Gamma \backslash \mathbb{H}^{2}$.

We are now in a position to construct point-pair invariants as in Definition 3 through

$$
\Phi(\sigma):=\frac{1}{\pi} \int_{-\infty}^{\infty} H(\sigma ; \rho) h(\rho) \mathrm{d} \rho
$$

where $h$ is a function as specified below.

Definition 5. A function $h: \mathbb{C} \rightarrow \mathbb{C}$ that satisfies

- $h$ is even, i.e. $h(\rho)=h(-\rho)$,

- $h$ is complex analytic in the strip $|\operatorname{Im}(\rho)| \leq \beta$ for some fixed $\beta \geq \frac{1}{2}+\epsilon$,

- there exists $\delta>0$, such that the bound

$$
|h(\rho)| \leq \text { const. }(1+\operatorname{Re}(\rho))^{-2-\delta}
$$

holds uniformly for all $\rho$ in the above mentioned strip,

is called an admissible test function.

We denote the Fourier transform of an admissible test function by

$$
g(u):=\frac{1}{2 \pi} \int_{-\infty}^{\infty} h(\rho) \mathrm{e}^{-\mathrm{i} \rho u} \mathrm{~d} \rho .
$$

All that we have to check that this indeed leads to a point-pair invariant is the condition (5.1). 
Lemma 7. Let $\sigma-1 \geq \kappa>0$, then the components $K_{i}$ of $K$ are bounded from above by

$$
\left|K_{i}\left(z^{\prime}, z\right)\right| \leq C_{\kappa} \mathrm{e}^{-\left(\frac{1}{2}+\beta\right) d\left(z^{\prime}, z\right)}, \quad i=1, \ldots, 4,
$$

with some $C_{\kappa}>0$. Furthermore, the limit $\lim _{d\left(z^{\prime}, z\right) \rightarrow 0} K\left(z^{\prime}, z\right)$ is well-defined. More precisely, we have

$$
\operatorname{tr} K(z, z)=\frac{1}{2 \pi} \int_{-\infty}^{\infty} \rho h(\rho) \operatorname{coth}(\pi \rho) \mathrm{d} \rho
$$

Proof. To prove the first part one shifts the integral by $-\mathrm{i} \beta$. Using the estimate (6.5) on $G_{i}$ and shifting back, the bound (6.6) follows immediately. For the second part we note that

$$
\lim _{\sigma \rightarrow 1^{+}} \frac{1}{4 \pi} \int_{-\infty}^{\infty} \rho h(\rho) \log (\sigma-1) \mathrm{d} \rho=0
$$

thus we can add this term to $\Phi$. Then again shifting the integral by $-\mathrm{i} \epsilon$ and using MOS66. p. 44] one obtains

$$
\lim _{d\left(z^{\prime}, z\right) \rightarrow 0} K_{i}\left(z^{\prime}, z\right)=\frac{1}{4 \mathrm{i} \pi^{2}} \int_{-\infty-\mathrm{i} \epsilon}^{\infty-\mathrm{i} \epsilon} \rho h(\rho)[\psi(\mathrm{i} \rho)+\psi(\mathrm{i} \rho+1)] \mathrm{d} \rho, \quad i=1,4,
$$

where $\psi(z)=\frac{\mathrm{d}}{\mathrm{d} z} \log \Gamma(z)$. After substituting $\rho$ by $-\rho$ and using the fact that $h$ is even the statement follows immediately.

Having showed that $K$ is indeed a point-pair invariant, we can introduce the automorphic kernel

$$
K_{\Gamma}\left(z^{\prime}, z\right):=\frac{1}{2} \sum_{\gamma \in \bar{\Gamma}} K\left(z^{\prime}, \gamma z\right) \chi(\gamma) J_{\gamma}(z, 1)
$$

which by construction is in $\mathfrak{L}^{2}\left(\Gamma \backslash \mathbb{H}^{2}, \Gamma \backslash \mathbb{H}^{2}, \chi\right)$. The corresponding Hilbert-Schmidt operator on $\mathfrak{L}^{2}(\Gamma, 1, \chi)$ is called $\mathrm{L}$.

Lemma 8. Any $\Psi \in \mathfrak{F}_{\rho}(\Gamma, 1, \chi)$ is simultaneously an eigenform of the Hilbert-Schmidt operator L,

$$
[\mathrm{L} \Psi]\left(z^{\prime}\right)=\Lambda(\rho) \Psi\left(z^{\prime}\right)
$$

where the eigenvalue $\Lambda$ satisfies the equation

$$
\Lambda(\rho)+\Lambda(-\rho)=2 h(\rho) .
$$

Proof. With

$$
[\mathrm{L} \psi]\left(z^{\prime}\right)=\frac{1}{2 \pi \mathrm{i}} \int_{F}\left(\sum_{\gamma \in \bar{\Gamma}} \int_{-\infty}^{\infty} h(\rho) G\left(z^{\prime}, \gamma z ; \rho\right) \chi(\gamma) J_{\gamma}(z, 1) \mathrm{d} \rho\right) \Psi(z) \mathrm{d} \mu(z)
$$


a standard calculation yields

$$
[\mathrm{L} \psi]\left(z^{\prime}\right)=\frac{1}{\pi \mathrm{i}} \int_{-\infty-\mathrm{i} \beta}^{\infty-\mathrm{i} \beta} h\left(\rho^{\prime}\right)\left(\int_{F} G_{\Gamma}\left(z^{\prime}, z ; \rho^{\prime}\right) \Psi(z) \mathrm{d} \mu(z)\right) \mathrm{d} \rho^{\prime} .
$$

From this we read off the eigenvalue of $\mathrm{L}$ as

$$
\Lambda(\rho)=\frac{1}{2 \pi \mathrm{i}} \int_{-\infty-\mathrm{i} \beta}^{\infty-\mathrm{i} \beta} \frac{h\left(\rho^{\prime}\right)}{\rho^{\prime}-\rho} \mathrm{d} \rho^{\prime}
$$

and this implies (6.8).

As already noted in Section 4, the spectrum of the Dirac operator on a compact surface is real and discrete. Moreover, according to Lemma 1 the spectrum is symmetric with respect to zero. We hence denote the non-negative eigenvalues (listed with their respective multiplicities) by $0 \leq \rho_{0} \leq \rho_{1} \leq \ldots$, which include $N$ of the $2 N$ zero modes. The Hilbert space $\mathfrak{L}^{2}(\Gamma, 1, \chi)$ therefore has a basis of orthonormal eigenforms $\Psi_{n, \pm}$,

$$
\left(\mathrm{D} \pm \rho_{n}\right) \Psi_{n, \pm}=0
$$

Thus the automorphic kernel possesses a spectral expansion of the form

$$
K_{\Gamma}\left(z^{\prime}, z\right)=\sum_{n=0}^{\infty}\left(\Lambda\left(\rho_{n}\right) \Psi_{n,+}\left(z^{\prime}\right) \Psi_{n,+}^{\dagger}(z)+\Lambda\left(-\rho_{n}\right) \Psi_{n,-}\left(z^{\prime}\right) \Psi_{n,-}^{\dagger}(z)\right)
$$

which immediately yields the spectral side of the desired trace formula.

Lemma 9. The Hilbert-Schmidt operator L has a finite trace, given by

$$
\operatorname{tr}(\mathrm{L})=2 \sum_{n=0}^{\infty} h\left(\rho_{n}\right)
$$

Next we need to compute the geometric side of the trace formula, i.e.,

$$
\operatorname{tr}(\mathrm{L})=\int_{F} K_{\Gamma}(z, z) \mathrm{d} \mu(z)
$$

with the representation (6.7) of the automorphic kernel. Following a standard procedure, this yields

$$
\operatorname{tr}(\mathrm{L})=\frac{1}{2} \sum_{\{\gamma\}} \chi(\gamma) \sum_{[g] \in \bar{Z}_{\gamma} \backslash \bar{\Gamma}} \operatorname{tr}\left(\int_{g F} K(z, \gamma z) J_{\gamma}(z, 1) \mathrm{d} \mu(z)\right)
$$


where $\{\gamma\}$ denotes the $\bar{\Gamma}$-conjugacy classes of $\gamma \in \bar{\Gamma}$ and $\bar{Z}_{\gamma}$ is the centraliser of $\gamma$ in $\bar{\Gamma}$. Next we use the natural pairing of the disjoint conjugacy classes $\{\gamma\}$ and $\{-\gamma\}$, which cancels the factor $\frac{1}{2}$. Within these pairs we choose the conjugacy classes with $\operatorname{tr}(\gamma)>0$. Moreover, it is well-known that

$$
D_{\gamma}:=\bigcup_{[g] \in Z_{\gamma} \backslash \Gamma} g(F)
$$

is a fundamental set for the centraliser $Z_{\gamma} \subset \Gamma$. Introducing primitive hyperbolic elements $\gamma_{p}$ and their conjugacy classes, one can rewrite (6.9) as

$$
\begin{aligned}
\operatorname{tr}(L)= & \operatorname{tr}\left(\int_{F} K(z, z) \mathrm{d} \mu(z)\right) \\
& +\sum_{\left\{\gamma_{p}\right\}} \sum_{n=1}^{\infty} \chi\left(\gamma_{p}^{n}\right) \operatorname{tr}\left(\int_{D_{\gamma_{p}}} K\left(z, \gamma_{p}^{n} z\right) J_{\gamma_{p}^{n}}(z, 1) \mathrm{d} \mu(z)\right) .
\end{aligned}
$$

Upon a conjugation with a matrix in $\mathrm{SL}(2, \mathbb{R})$ any hyperbolic element can be brought into the Jordan normal form $\gamma=\left(\begin{array}{cc}\mathrm{e}^{\frac{l_{\gamma}}{2}} & 0 \\ 0 & \mathrm{e}^{-\frac{l_{\gamma}}{2}}\end{array}\right)$. A fundamental domain for the group generated by $\gamma$ is given by $\left\{z \in \mathbb{H}^{2} \mid 1<y<\mathrm{e}^{l_{\gamma}}\right\}$. Note that $J_{\gamma}(z, 1)=1$, so all that remains to be done is evaluating integrals of the form

$$
I\left(\gamma^{n}\right)=\operatorname{tr}\left(\int_{1}^{\mathrm{e}^{l}} \int_{-\infty}^{\infty} K\left(z, \gamma^{n} z\right) \mathrm{d} x \frac{\mathrm{d} y}{y^{2}}\right) .
$$

In order to calculate this integral we introduce

$$
\tau=\cosh ^{2} \frac{n l}{2}+\left(\frac{x}{y} \sinh \frac{n l}{2}\right)^{2},
$$

which allows us to perform the integration with respect to $y$. Inserting the explicit expressions (6.3) and (6.4) for the function $H$, the integral (6.10) reduces to

$$
\begin{aligned}
I\left(\gamma^{n}\right)= & \frac{\mathrm{i} l_{\gamma}}{2 \pi^{2}} \operatorname{coth} \frac{n l_{\gamma}}{2} \int_{-\infty}^{\infty} \rho h(\rho) \times \\
& \times \int_{\cosh ^{2} \frac{n l_{\gamma}}{2}}^{\infty} \tau^{-1-\mathrm{i} \rho}\left(\tau-\cosh ^{2} \frac{n l_{\gamma}}{2}\right)^{-\frac{1}{2}} \sum_{m=0}^{\infty} \frac{\Gamma(\mathrm{i} \rho+m) \Gamma(\mathrm{i} \rho+m+1)}{\Gamma(2 \mathrm{i} \rho+m+1) m !} \tau^{-m} \mathrm{~d} \tau \mathrm{d} \rho .
\end{aligned}
$$

Interchanging the order of integration and summation and employing the relation

$$
\left(\frac{1}{2 \cosh \left(\frac{a}{2}\right)}\right)^{-2 q} \sum_{m=0}^{\infty} \frac{2 q \Gamma(2 q+2 m)}{\Gamma(2 q+m+1) m !}\left(\frac{1}{2 \cosh \left(\frac{a}{2}\right)}\right)^{-2 m}=\mathrm{e}^{-q a}
$$


which follows from [GR65, 1.114] for $a>0$ and $\operatorname{Re}(q)>0$, finally yields

$$
I\left(\gamma^{n}\right)=\frac{l_{\gamma}}{\sinh \frac{n l_{\gamma}}{2}} \frac{1}{2 \pi} \int_{-\infty}^{\infty} h(\rho) \mathrm{e}^{-\mathrm{i} \rho n l_{\gamma}} \mathrm{d} \rho=\frac{l_{\gamma} g\left(n l_{\gamma}\right)}{\sinh \left(\frac{n l_{\gamma}}{2}\right)} .
$$

Putting together Lemma [7. Lemma 9 and equation (6.11) then leads to the desired trace formula.

Theorem 1 (Selberg trace formula for the Dirac operator). Let $\Gamma \subset \operatorname{PSL}(2, \mathbb{R})$ be a strictly hyperbolic Fuchsian group with fundamental domain $F$ of area $A(F)$ and fix a multiplier system $\chi$ of weight one. Moreover, let $\left\{\rho_{n}\right\}_{n=0}^{\infty}$ be the non-negative eigenvalues of the Dirac operator $\mathrm{D}$ on $\mathfrak{L}^{2}(\Gamma, 1, \chi)$, including half of the zero-modes. Then, for any admissible test function $h$, see Definition 5, the following trace formula holds,

$$
\sum_{m=0}^{\infty} h\left(\rho_{m}\right)=\frac{A(F)}{4 \pi} \int_{-\infty}^{\infty} \rho h(\rho) \operatorname{coth}(\pi \rho) \mathrm{d} \rho+\sum_{\left\{\gamma_{p}\right\}} \sum_{n=1}^{\infty} \chi\left(\gamma_{p}^{n}\right) \frac{l_{\gamma_{p}} g\left(n l_{\gamma_{p}}\right)}{2 \sinh \left(\frac{n l_{\gamma_{p}}}{2}\right)} .
$$

In accordance with Proposition 11 this trace formula is identical to the one for the Maaß-Laplacian $-\Delta_{1}$ on the same surface and with the same multiplier system. We stress, however, a difference in the interpretation of the geometric side. Both expressions can be viewed as sums over the closed geodesics on $\Gamma \backslash \mathbb{H}^{2}$, weighted with the factors $\chi\left(\gamma_{p}^{n}\right)$. For the Maaß-Laplacian these factors stem from the non-vanishing magnetic fluxes that are necessarily present (see, e.g., CGO93]), whereas for the Dirac operator with weight $k=1$ there is no magnetic field involved. Here the factors $\chi\left(\gamma_{p}^{n}\right)$ reflect the fact that the classical dynamical system associated with the quantum dynamics generated by the Dirac operator is not the geodesic flow, but its associated frame flow, see also [JS06. This is analogous to Dirac operators on $\mathbb{R}^{3}$ BK99].

The frame flow (see, e.g., [BG80]) is a flow in the frame bundle $F M$ over a Riemannian manifold $M$ consisting of a parallel transport of oriented orthonormal frames along geodesics. To be precise, let $\left\{e_{1}(p), \ldots, e_{n}(p)\right\}$ be an orthonormal basis of $T_{p} M$. Then this frame is transported along the geodesic determined by $e_{1}$ with the Levi-Civita connection. This flow can be lifted into the spin structure by assigning an element $g(p) \in \operatorname{Spin}(n)$ to every point along the geodesic. This may also be done in the spin representation yielding $\rho(g(p))$. In the present case, where $M=\Gamma \backslash \mathbb{H}^{2}, \rho(g(z))$ must equal $\rho(g(\gamma z))$ for every $\gamma \in \bar{\Gamma}$. However, in the induced (trivial) bundle over the hyperbolic plane the transformation property

$$
\rho(g(\gamma z))=\chi(\gamma) J_{\gamma}(z, 1) \rho(g(z))
$$

applies, see (3.1) and (3.3). The factors $\chi\left(\gamma_{p}^{n}\right)$ in equation (6.12) therefore reflect the spin structure. Hence, although on two-dimensional manifolds the frame flow is not much different from the geodesic flow, only the latter is a natural dynamics that can be lifted into the spin structure. For $n \geq 3$ the situation will be different since in such a case a frame flow yields interesting classical dynamics beyond the geodesic flow. 


\section{Some Applications}

A first immediate application of the trace formula, which can be proved in the standard way (see, e.g., Hej76), concerns the asymptotic distribution of the eigenvalues.

Proposition 3 (Weyl's law). Let $N(\rho)$ be the number of non-negative eigenvalues of $D$ which are smaller than $\rho$. Then

$$
N(\rho) \sim \frac{A(F)}{4 \pi} \rho^{2}, \quad \rho \rightarrow \infty .
$$

Another application consists of determining properties of the related Selberg zeta function. If $\operatorname{Re}(s), \operatorname{Re}(\sigma)>1$ the function

$$
h(\rho):=\frac{1}{\rho^{2}+\left(s-\frac{1}{2}\right)^{2}}-\frac{1}{\rho^{2}+\left(\sigma-\frac{1}{2}\right)^{2}}
$$

satisfies the criteria of Definition 5 to serve as an admissible test function. In the trace formula (6.12) it leads to a relation for the trace of a regularised resolvent,

$$
\begin{aligned}
\sum_{m=0}^{\infty}\left(\frac{1}{\rho_{m}^{2}+\left(s-\frac{1}{2}\right)^{2}}-\frac{1}{\rho_{m}^{2}+\left(\sigma-\frac{1}{2}\right)^{2}}\right)= & -\frac{A(F)}{2 \pi}\left(\psi\left(s-\frac{1}{2}\right)-\psi\left(\sigma-\frac{1}{2}\right)\right) \\
& +\frac{A(F)}{4 \pi}\left(\frac{1}{\sigma-\frac{1}{2}}-\frac{1}{s-\frac{1}{2}}\right) \\
& +\frac{1}{2 s-1} \frac{Z^{\prime}(s)}{Z(s)}-\frac{1}{2 \sigma-1} \frac{Z^{\prime}(\sigma)}{Z(\sigma)} .
\end{aligned}
$$

Here $Z(s)$ is Selberg's zeta function which is defined by

$$
Z(s):=\prod_{\left\{\gamma_{p}\right\}} \prod_{k=0}^{\infty}\left(1-\chi\left(\gamma_{p}\right) \mathrm{e}^{-l_{\gamma_{p}}(k+s)}\right), \quad \operatorname{Re}(s)>1 .
$$

Now proceeding along the lines of [Ste87, Sar87] we find an analytic continuation of $Z(s)$ into the entire complex plane.

Proposition 4. The Selberg zeta function for the Dirac operator is an entire analytic function. Moreover, it can be represented as

$$
\begin{aligned}
Z(s)= & \frac{Z^{(2 N)}\left(\frac{1}{2}\right)}{(2 N) !}\left(s-\frac{1}{2}\right)^{2 N} \mathrm{e}^{\left(s-\frac{1}{2}\right)^{2} \gamma_{D}} \mathrm{e}^{\left(s-\frac{1}{2}\right) \frac{A(F)}{2 \pi}} \times \\
& \times\left[(2 \pi)^{-\left(s-\frac{1}{2}\right)} \mathrm{e}^{\left(s^{2}-\frac{1}{4}\right)} G^{2}\left(s+\frac{1}{2}\right)\right]^{\frac{A(F)}{2 \pi}} \prod_{m=N}^{\infty}\left[\left(1+\frac{\left(s-\frac{1}{2}\right)^{2}}{\rho_{m}^{2}}\right) \mathrm{e}^{-\frac{\left(s-\frac{1}{2}\right)^{2}}{\rho_{m}^{2}}}\right],
\end{aligned}
$$

where $G$ is Barnes' double $\Gamma$-function (see [Bar99]) and $2 N$ denotes the number of zero modes of $\mathrm{D}$. The trivial zeroes of $Z(s)$ are given by $s=-\frac{1}{2}-n$ with multiplicities $(2 n)^{\frac{A(F)}{2 \pi}}$, $n \in \mathbb{N}$. The non-trivial zeroes are $s=\frac{1}{2} \pm \mathrm{i} \rho_{m}$, with the same multiplicities as the eigenvalues $\rho_{m}$ of $\mathrm{D}$. 
In (17.1) the constant $\gamma_{D}$ is a generalised Euler constant as introduced in [Ste87] and $Z^{(2 N)}$ denotes a derivative of the zeta function of order $2 N$. Moreover, due to the Gauß-

Bonnet theorem, $\frac{A(F)}{2 \pi}=2(g-1)$ is a positive integer, where $g$ is the genus of the surface $\Gamma \backslash \mathbb{H}^{2}$.

\section{References}

[AS68] M.F. Atiyah and I.M. Singer, The index of elliptic operators, Ann. of Math. 87 (1968), 546-604.

[Bar99] E.W. Barnes, The theory of the G-function, Quart. J. Math. 31 (1899), 264-314.

[Bau81] H. Baum, Spin-Strukturen und Dirac-Operatoren über Pseudo-Riemannschen Mannigfaltigkeiten, Teubner-Verlag Leipzig, 1981.

[BG80] M. Brin and M. Gromov, On the ergodicity of frame flows, Invent. Math. 60 (1980), $1-7$.

[BGGS92] E. Bogomolny, B. Georgeot, M. J. Giannoni, and C. Scmmit, Chaotic billiards generated by arithmetic groups, Phys. Rev. Lett. 69 (1992), 1477-1480.

[BGS84] O. Bohigas, M. J. Giannoni, and C. Schmit, Characterization of chaotic quantum spectra and universality of level fluctuation laws, Phys. Rev. Lett. 52 (1984), 1-4.

[BH03] J. Bolte and J. M. Harrison, Spectral statistics for the Dirac operator on graphs, J. Phys. A: Math. Gen. 36 (2003), 2747-2769.

[BK99] J. Bolte and S. Keppeler, A semiclassical approach to the Dirac equation, Ann. Phys. (NY) 274 (1999), 125-162.

[BO95] U. Bunke and M. Olbrich, Selberg zeta and theta functions, Akademie Verlag, 1995.

[BSS92] J. Bolte, G. Steil, and F. Steiner, Arithmetical chaos and violation of universality in energy level statistics, Phys. Rev. Lett. 69 (1992), 2188-2191.

[CGO93] A. Comtet, B. Georgeot, and S. Ouvry, Trace formula for Riemann surfaces with magnetic field, Phys. Rev. Lett. 71 (1993), 3786-3789.

[Erd53] A. Erdélyi, Higher transcendental functions, vol. 1, Mc Graw-Hill, 1953.

[Fri97] T. Friedrich, Dirac-Operatoren in der Riemannschen Geometrie, Vieweg Verlag Braunschweig, 1997.

[GR65] I.S. Gradshteyn and I.M. Ryzhik, Table of integrals, series and products, 4th ed., Academic Press, 1965.

[Gun56] R.C. Gunning, The structure of factors of automorphy, Amer. J. Math. 78 (1956), $357-382$.

[Gun76]__ Riemann surfaces and generalized theta functions, Springer-Verlag, 1976. 
[Hej76] D.A. Hejhal, The Selberg trace formula for PSL(2, R), vol. 1, Springer-Verlag, 1976.

[Hej83] _ The Selberg trace formula for $\operatorname{PSL}(2, \mathbb{R})$, vol. 2, Springer-Verlag, 1983.

[Hit74] N. Hitchin, Harmonic spinors, Adv. in Mathematics 14 (1974), 1-55.

[JS06] D. Jakobson and A. Strohmaier, High energy limits of Laplace-type and Dirac-type eigenfunctions and frame flows, preprint, 2006.

[Kra30] H. Kramers, Théorie générale de la rotation paramagnétique dans le cristaux, Proc. Acad. Amst. 33 (1930), 959-972.

[Maa53] H. Maaß, Die Differentialgleichungen in der Theorie der elliptischen Modulfunktionen, Math. Ann. 125 (1953), 235-263.

[MOS66] W. Magnus, F. Oberhettinger, and R.P. Soni, Formulas and theorems for the special functions of mathematical physics, 3rd ed., Springer-Verlag, 1966.

[Pnu94] A. Pnueli, Spinors and scalars on Riemann surfaces, J. Phys. A: Math. Gen. 27 (1994), 1345-1352.

[Roe66] W. Roelcke, Das Eigenwertproblem der automorphen Formen in der hyperbolischen Ebene I, Math. Ann. 167 (1966), 292-337.

[Roe67] _ Das Eigenwertproblem der automorphen Formen in der hyperbolischen Ebene II, Math. Ann. 168 (1967), 261-324.

[Sar87] P. Sarnak, Determinants of Laplacians, Commun. Math. Phys. 110 (1987), 113-120.

[Sel56] A. Selberg, Harmonic analysis and discontinuous groups in weakly symmetric Riemannian spaces with applications to Dirichlet series, J. Indian Math. Soc. 20 (1956), $47-87$.

[Ste87] F. Steiner, On Selberg's zeta function for Riemann surfaces, Phys. Lett. 188B (1987), $447-454$.

[Tha92] B. Thaller, The Dirac Equation, Texts and Monographs in Physics, Springer-Verlag, Berlin, 1992.

[Zir96] M. Zirnbauer, Riemannian symmetric superspaces and their origin in random matrix theory, J. Math. Phys. 37 (1996), 4986-5018. 